\title{
Análisis estadístico de la elección presidencial 2014 en Costa Rica
}

\author{
Statistical analysis of the 2014 presidential election of Costa Rica
}

\author{
Carlomagno Araya Alpízar ${ }^{1}$ \\ Recibido: 13/4/2016 / Aprobado: 7/3/2017
}

\begin{abstract}
Resumen
En el presente trabajo se analiza el impacto de las condiciones económicas, y demográficas distritales y cantonales, sobre los resultados de la elección presidencial de Costa Rica en el año 2014. La evidencia estadística muestra como variables relevantes para explicar los resultados electorales las siguientes: tasa neta de participación, tasa de ocupación, porcentaje de población urbana, tasa de fecundidad general e índice de desarrollo social. Como principal resultado se obtuvo que las regiones con mayor tasa de participación y tasa de ocupación tendieron a apoyar al Partido Acción Ciudadana.
\end{abstract}

Palabras claves: Costa Rica, elección, presidente, Biplot.

Abstract

In this paper the impact of economic conditions and demographic district and cantonal regions is analyzed. It is based on results population elections held in 2014 Costa Rica presidential election. The evidence shows relevant variables that explain the voting as: net participation rate, employment rate, urban population percentage, fertility rate and social development index. One important detail is that the highest participation and employment rate come from the population that tends to support Partido Acción Ciudadana.

Key words: Costa Rica, election, president, Biplot.

\section{Introducción}

En este trabajo se analiza el efecto de las condiciones económicas y demográficas de los distritos y cantones sobre el resultado de la elección presidencial en Costa Rica del año 2014, al respecto Moreno (2009) menciona: "En este sentido, los factores típicos de un modelo convencional de decisión de voto, incluyen la identificación partidista del votante; la imagen de los candidatos; las evaluaciones económicas; las posturas en los temas políticos relevantes y de campaña, las características ideológicas, socio demográficas y geográficas de los votantes" (p. 277).

El abordaje estadístico de las elecciones permitió identificar las variables que caracterizan los distritos y cantones de Costa Rica que influyen estadísticamente en el resultado de las elecciones para la elección del presidente. Dado que la

\footnotetext{
${ }^{1}$ Magister en Estadística, Universidad de Costa Rica, doctor en Estadística Multivariada Aplicada, Universidad de Salamanca, España y bachiller en Ciencias Políticas, Universidad de Costa Rica. Profesor catedrático de Estadística, Departamento de Ciencias Naturales, Universidad de Costa Rica. Correo electrónico: carlo.araya@ucr.ac.cr / carlomagno@gmx.com
} 
situación económica no afecta uniformemente a todas las regiones del país, debe considerarse que los resultados de las urnas no responden únicamente a las campañas electorales, cualidades de los candidatos y partidos políticos, sino que involucran una compleja mezcla de condiciones económicas y características demográficas imperantes en Costa Rica.

En este sentido, Constantino Urcuyo (2015) manifiesta que el resultado de las elecciones se debe a múltiple factores como: la ruptura del bipartismo presidencial, la decepción acumulada por muchos años con los partidos y con el sistema democrático. También, por la pérdida de credibilidad del gobierno de Doña Laura Chinchilla, "que no cometió grandes errores pero que navegó al garete sin hoja de ruta definida”. El votante castiga o premia el desempeño del partido político del gobierno de turno con miras a mejorar su situación económica. Además, hay algunos analistas políticos que consideran que el voto tiene un componente de "inercia"; los electores tienden a votar por el candidato que se ubica en el mismo nivel socioeconómico.

En cuanto a los métodos estadísticos utilizados en este trabajo, se encuentran "los métodos Biplot que se usan para representar conjuntos de datos multivariados, contenidos en una matriz $\mathbf{X}_{\mathbf{n p}}$ sin hacer supuestos sobre modelos subyacentes ni distribuciones poblacionales. De igual manera que un diagrama de dispersión muestra la distribución conjunta de dos variables (X, Y), un Biplot representa tres o más variables en un espacio de dimensión reducida" (Gabriel y Odoroff,1990, citado en Araya, 2015).

El desarrollo del trabajo se realizó en las siguientes secciones: en la primera etapa, la metodología y más específicamente, el método Biplot y las fuentes de los datos. A continuación, se analizan los resultados usando métodos estadísticos univariados y multivariados. En la última etapa se presenta las conclusiones estadísticas del resultado de las elecciones para presidente.

\section{Metodología}

Los métodos Biplot son técnicas de análisis multivariados que buscan reducir la dimensión de una matriz $\mathbf{X}_{(\mathbf{n x p})}$ a través de la proyección de los datos originales en un subespacio de extensión reducida que conserva los patrones fundamentales, respecto de la variación conjunta de los individuos (filas) y las variables (columnas).

$$
\boldsymbol{X}_{n x p}=\left(\begin{array}{ccc}
X_{11} & \ldots & X_{1 p} \\
\vdots & X_{i j} & \vdots \\
X_{n 1} & \ldots & X_{n p}
\end{array}\right)
$$

La matriz $\mathbf{X}$ de orden (nxp) y rango $\mathbf{r}$, es aproximada por otra de rango $\mathbf{q}$ tal que $\boldsymbol{q}<\boldsymbol{r}$, usando una descomposición en los valores singulares (DVS):

$$
X \cong U \Sigma_{\alpha} V^{T}
$$

donde $\mathbf{U}$ y $\mathbf{V}$ son matrices de vectores singulares ortonormales, tal que $\mathbf{U}^{\mathrm{T}} \mathbf{U}=\mathbf{V}^{\mathrm{T}} \mathbf{V}=\mathbf{I}$ (I es la matriz identidad) y $\Sigma_{\alpha}$ es una matriz diagonal que contienes $\operatorname{los} \alpha_{k}$ valores singulares. Entonces, la matriz $\mathbf{Y}$ se puede descomponer como:

$$
X=F C^{T}
$$

para $\mathbf{F}=\mathbf{U} \boldsymbol{\Sigma}_{\text {s }}^{\boldsymbol{\alpha}}$ y $\mathbf{C}=\mathbf{V} \boldsymbol{\Sigma}_{\boldsymbol{\alpha}}^{\mathbf{1} \text { s }}$. Las matrices $\mathbf{F}$ y $\mathbf{C}$ están formadas por las coordenadas de los marcadores filas (vectores fila $\boldsymbol{f}_{i}$ ) y columnas (vectores columna $\boldsymbol{c}_{\boldsymbol{j}}$, utilizados para la representación gráfica Biplot.

La elección de distintas métricas hará posible que la representación tenga diferentes propiedades, por lo cual la escogencia de métricas distintas puede ayudar a poner de manifiesto diversos aspectos relevantes de los datos. En el JK-Biplot se aproxima la matriz $\mathrm{X}$ bajo la condición que $\boldsymbol{V}^{T} \boldsymbol{V}=\boldsymbol{I}$, siendo los marcadores filas los resaltantes ya que preservan la métrica entre las filas. En tanto, GH-Biplot se aproxima $\mathbf{X}$ siendo los marcadores columnas representados con mayor calidad de representación. El HJ-Biplot es una alternativa para obtener igual calidad de representación para filas y columnas, 
usando la factorización $\mathbf{X}=\mathbf{U D V}^{\mathrm{T}}, \mathrm{J}=\mathrm{UD}, \mathrm{H}=\mathrm{VD}$. Sin embargo, este tipo de Biplot tiene la desventaja que no permite reproducir la matriz original de datos.

Cuando se realiza una aproximación de un espacio n-dimensional, por medio de un subespacio de menor dimensión se tiene una pérdida de información, por lo tanto, se hace necesario valorar la bondad del ajuste de la nueva representación, para ello se utiliza como estimador el estadístico de absorción de inercia, de manera que cuanto más se acerque el valor al 100\% tendrá mayor confiabilidad (Cárdenas et al, 2007 citado en Araya, 2015).

$\mathrm{Al}$ interpretar una representación Biplot se debe tener presentes unas referencias básicas, el segmento o vector desde el origen representa el valor de la desviación típica de la variable, y para interpretarlo se debe tener en cuenta que: su dirección muestra los valores crecientes de la variable; la longitud del vector representa la variabilidad (desviación típica) que habrá presentado la variable en el estudio, de forma que la longitud es un indicador importante en el análisis de los resultados.

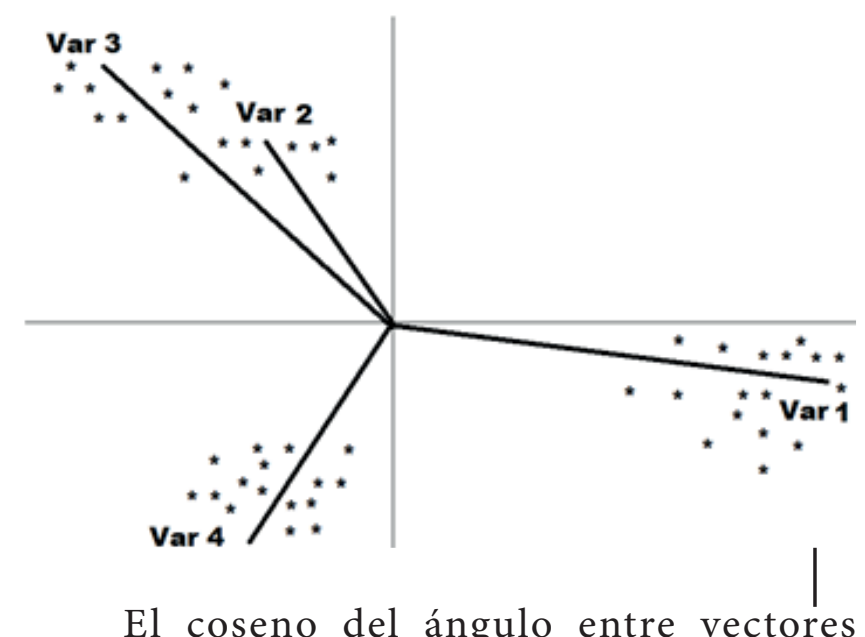
representativos de las variables, refleja el grado de asociación entre ellas, como consecuencia; cuanto menor sea el ángulo entre dos vectores que unen el centro de gravedad con los puntos que representan a las variables, más correlación entre sí.
La proyección de un individuo en la dirección de una variable permite conocer el valor de esta para el individuo. La proyección de una variable sobre un individuo indica la importancia de la variable en la representación del sujeto. Si entre una variable y un individuo existe un ángulo muy pequeño, significa que el individuo es significativo para explicar la variable y que esta tiene un gran valor para él.

\section{Recolección de los datos}

Los datos para realizar el trabajo de investigación se obtuvieron de tres fuentes primarias. Los resultados de las elecciones se tomaron del Tribunal Supremo de Elecciones de Costa Rica, según cómputo de votos definitivos de las elecciones para Presidente de 2014 por distrito y por cantón.

Con respecto a las características demográficas y socioeconómicas de los distritos se obtuvieron del Instituto Nacional de Estadística y Censos según los resultados de Censo de Población y Vivienda de 2011. La tercera fuente de información fue el Ministerio de Planificación Nacional y Política Económica, donde se consiguieron los datos sobre la clasificación de los distritos y cantones de Costa Rica según el Índice de Desarrollo Social de 2013.

Las variables socio-demográficas se describen a continuación:

- Relación de dependencia demográfica (RDD). Es la relación existente entre la población dependiente y la población productiva, de la que aquella depende.

- Relación de dependencia infantil (RDI). Es la relación entre el número de niños (as) entre 0-14 años y las personas entre 15-64 años (activas).

- Porcentaje de población urbana (PPU). Las personas que viven en zonas urbanas.

- Tasa neta de participación (TNP). Es el porcentaje de la fuerza de trabajo con respecto a la población en edad de trabajar. 
- Tasa de ocupación (TDO). Es el porcentaje de la población ocupada con respecto a la población en edad de trabajar.

- Tasa de desempleo abierto (TDA). Es el porcentaje de la población que no trabajó en la semana de referencia y lo buscó en las cinco semanas anteriores, con relación a la fuerza de trabajo. Incluye cesantes y los que buscan trabajo por primera vez.

- Relación de dependencia económica (RDE). Relación entre las personas inactivas y las activas.

El Índice de Desarrollo Social (IDS), es usado para clasificar los distritos y cantones del país de acuerdo con su nivel de desarrollo social. Se construye a partir de un conjunto de 11 índices socioeconómicos: educación (infraestructura educativa, programas educativos especiales, escuelas unidocentes y reprobación escolar), participación electoral, salud (bajo peso en niños(as), calidad de agua potable residencial, nacimientos en madres adolescentes solteras), economía (viviendas con acceso a internet, consumo residencial de electricidad) (MIDEPLAN, 2013).

El análisis se realizó a través del programa MultBiplot desarrollado por Vicente-Villardón (2010) en el sistema Matlab. Este es un lenguaje de alto nivel y un entorno interactivo para el cálculo numérico, la visualización y la programación orientado a matrices.

\section{Análisis de Resultados}

Primeramente, es posible notar una relación estadística entre el nivel de desarrollo de los distritos y el porcentaje de participación en las elecciones de
2014 (Gráfico 1). Esto implica que ante menor nivel de desarrollo social, el porcentaje de participación tiende a decrecer. Esta situación muestra que es posible ajustar un modelo de regresión lineal simple, para predecir el porcentaje de participación usando como variable independiente (explicativa) el nivel de desarrollo social de los distritos.

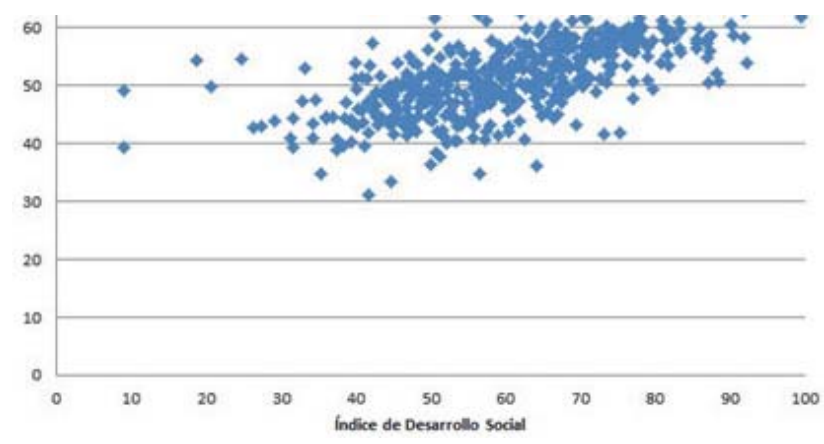

Gráfico 1. Representación de la relación entre el Índice de Desarrollo Social de los distritos y el porcentaje de participación en las elecciones de 2014.

En este sentido, el número de votos obtenidos por el Partido Acción Ciudadana (PAC), presentan la mayor asociación positiva con las variables Índice de Desarrollo Social y porcentaje de población urbana, el coeficiente de correlación lineal de Pearson $^{2}$ fueron de 0,42 y 0,54 respectivamente.

En tanto las correlaciones de los votos obtenidos por el Partido Liberación Nacional (PLN), son significativamente inferiores a las conseguidas por el PAC, esto un indicador que las regiones que votaron por el PLN tienen un menor IDS. Solamente el partido Renovación Costarricense (RC) tiene una correlación negativa con el IDS. Lo que implica que los votos favorables a RC decrecen conforme el IDS aumenta (Cuadro 1).

\footnotetext{
2 El coeficiente de correlación de Pearson es una medida estadística de la asociación lineal entre dos variables aleatorias cuantitativas. Se calcula como:
}

$$
r=\frac{n \sum x y-\sum x \sum y}{\sqrt{\left[n \sum x^{2}-\left(\sum x\right)^{2}\right]\left[n \sum y^{2}-\left(\sum y\right)^{2}\right]}}
$$


Cuadro 1. Coeficiente de correlación de Pearson entre las variables de estudio y los votos obtenidos por los partidos políticos, Elecciones de 2014.

\begin{tabular}{|c|c|c|c|c|c|c|c|c|c|c|c|c|c|}
\hline PARTIDO & PAC & PLN & FA & ML & USC & ASE & AN & PT & IN & NG & PN & RC & RN \\
\hline IDS & 0,42 & 0,21 & 0,14 & 0,16 & 0,14 & 0,12 & 0,19 & 0,11 & 0,25 & 0,29 & 0,22 & $-0,21$ & 0,10 \\
\hline PPU & 0,54 & 0,39 & 0,35 & 0,41 & 0,38 & 0,38 & 0,41 & 0,35 & 0,44 & 0,50 & 0,32 & 0,07 & 0,34 \\
\hline TNP & 0,47 & 0,25 & 0,22 & 0,30 & 0,22 & 0,26 & 0,28 & 0,25 & 0,33 & 0,38 & 0,29 & $-0,01$ & 0,22 \\
\hline TDO & 0,45 & 0,23 & 0,19 & 0,27 & 0,19 & 0,23 & 0,25 & 0,22 & 0,30 & 0,35 & 0,28 & $-0,49$ & 0,19 \\
\hline PPEI & $-0,47$ & $-0,25$ & $-0,22$ & $-0,30$ & $-0,22$ & $-0,26$ & $-0,28$ & $-0,25$ & $-0,33$ & $-0,38$ & $-0,29$ & 0,01 & $-0,22$ \\
\hline $\mathrm{RDE}$ & $-0,50$ & $-0,27$ & $-0,22$ & $-0,26$ & $-0,21$ & $-0,24$ & $-0,28$ & $-0,23$ & $-0,33$ & $-0,38$ & $-0,29$ & 0,11 & $-0,19$ \\
\hline
\end{tabular}

En general, la variable económica tasa neta de participación (TNP) y tasa de ocupación (TDO), presentaron una correlación positiva, mientras tanto la proporción de la población inactiva (PPEI) y la relación de dependencia económica (RDE), tienen asociación negativa, en ambos casos con el número de votos que obtuvieron los partidos políticos. En particular, el PAC presentó el mayor grado de correlación positiva con la tasa neta de participación $(r=0,47)$ y tasa de ocupación $(r=0,45)$. Implicando que regiones con mayores tasas de participación y tasa de ocupación tendieron a apoyar al PAC.

Es posible denotar como de la cantidad de votos favorables obtenidos por los partidos, fueron menores conforme aumentaba el porcentaje de la población inactiva y la relación de dependencia económica de los habitantes de los cantones.

Por otra parte, el análisis del resultado de la elección usando el método Biplot, muestra que la bondad en el ajuste global para el primer plano factorial (ejes 1 y 2) es 95\% (Gráfico 2). El plano factorial consiste en la representación gráfica de las coordenadas de cada elemento de las filas (distritos) y columnas (partidos políticos) en el espacio de factores. Las mayores calidades de representación acumuladas son del Partido Acción Ciudadana (PAC) y Partido Liberación Nacional (PLN), en tanto el Frente Amplio (FA) está mejor representado en el tercer eje factorial.

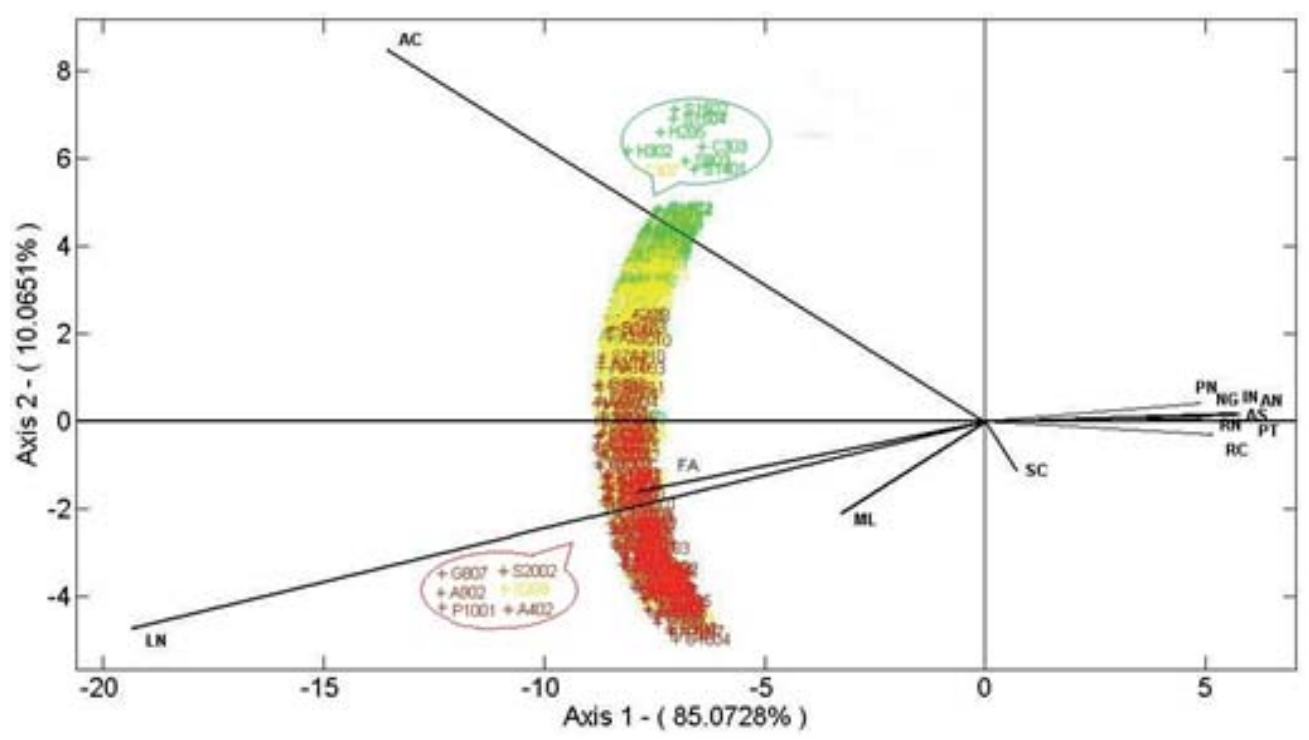

Gráfico 2. Representación de las elecciones Presidenciales según partido político e Índice de Desarrollo Social de los distritos. 
En el gráfico se representan los distritos según las siguientes categorías (o colores) de Índice de Desarrollo Social (IDS): verde incluye los distritos dentro del intervalo de 70 hasta 100 (mayor desarrollo relativo), amarillo son los distritos ubicados entre 60 y menos 70 (nivel medio) y rojo, representa a todos los distritos con niveles del IDS menores al 60 (nivel bajo).

Los resultados de las elecciones de 2014 muestran que el Partido Acción Ciudadana (PAC) ganó 186 (38,9\%) distritos, el Partido Liberación Nacional (PLN) ganó 290 (60,7\%) y Frente Amplio resultó vencedor en 2 distritos.

Es importante observar en el Gráfico 2, que el Partido Acción Ciudadana (PAC) tendió a ganar los distritos con mayores IDS, ejemplo: Sabanilla (S1502), San Rafael (S1504), Santa Lucía (H205), San Vicente (H302), San Juan (C303), Calle Blancos (S803), San Vicente (S1401) y San Ramón (C307). En tanto, el Partido Liberación Nacional (PLN) ganó en aquellos distritos con bajos niveles de desarrollo social, ejemplo: Arenal (G807), San Andrés (S2002), El Mastate (A902), Corredor (P1001), Desmonte (A402) y Rosario (S309). Con el propósito de determinar la provincia de pertenencia de los distritos, debe considerarse que la letra de los códigos representa la provincia. Por ejemplo, el distrito Mastate con código A902 se encuentra ubicado en la provincia de Alajuela.

Los resultados anteriormente citados, nos permiten explicar la hipótesis de algunos analistas políticos, que afirmaron a través de los medios de comunicación, que el PAC ganó en zonas urbanas y PLN en las zonas costeras. Lo que realmente ocurre y que afecta el resultado en un distrito es el Índice de Desarrollo Social (IDS) y no directamente que sea un distrito costero. En las zonas costeras coincidentemente existe nivel bajo IDS, en tanto, en los distritos de las zonas urbanas hay mayor desarrollo social. En los distritos ganados por el PAC el IDS promedio es 70,89, con una desviación estándar de 9,57 puntos, en tanto, los distritos que favorecieron al PLN la media aritmética del IDS es 52, 88 y una desviación estándar de 11,87 puntos.

Además, se encontraron diferencias estadísticas entre los distritos ganados por ambos partidos según variables demográficas y económicas. El Partido Acción Ciudadana obtuvo la mayor cantidad de votos a favor en los distritos con las siguientes características: tienen mayor porcentaje de población urbana $(81,3)$, la tasa neta de participación es 55,6 y la tasa de ocupación es 54,0. El Partido Liberación ganó los distritos con mayores problemas socioeconómicos, caracterizados por una relación de dependencia demográfica y de dependencia infantil de 52 y 41 respectivamente. Además, los distritos tienen mayores tasas de desempleo abierto, un porcentaje de población económicamente inactiva del 51,7 y la relación de dependencia económica es 1,9 (Cuadro 2).

Seguidamente, se analizarán resultados de elecciones Presidenciales por cantones. El Partido Acción Ciudadana (PAC), ganó 36 cantones y el Partido Liberación Nacional (PLN) en 45. La representación Biplot de los resultados por partidos políticos y cantones, tiene una bondad en el ajuste global para el primer plano factorial (ejes 1 y 2) de 97\% (Gráfico 3). Los cantones con mayores Índices de Desarrollo Social (IDS) tendieron a votar por el PAC y el PLN ganó en aquellos con menores IDS. La media aritmética del IDS es 71,6 en cantones ganados por el PAC y 39,7 en aquellos que favorecieron al PLN.

A manera de ejemplo, los cantones de Moravia (S14), Vásquez de Coronado (S11), Barva (H02), Santo Domingo (H03), Atenas (A05), Heredia (H01) y San Pablo (H09), fueron ganados por el Partido Acción Ciudadana (PAC). En contraposición, el Partido Liberación Nacional (PLN) ganó en Coto Brus (P08), Liberia (G01), León Cortés (S20), Turrubares (S16), Aguirre (P06), Abangares (G07) y Guácimo (L06). 
Cuadro 2. Comparación de los promedios aritméticos de las variables socioeconómicas según distritos ganados por el Partido Acción Ciudadana (PAC) y Partido Liberación Nacional

\begin{tabular}{|c|c|c|c|}
\hline Variable & & Media & $\begin{array}{c}\text { Desviación } \\
\text { estándar }\end{array}$ \\
\hline \multirow{2}{*}{ Relación de dependencia demográfica } & PAC & 43,95 & 4,03 \\
\hline & PLN & 52,00 & 6,49 \\
\hline \multirow{2}{*}{ Relación de dependencia infantil } & PAC & 32,58 & 5,71 \\
\hline & PLN & 41,05 & 7,57 \\
\hline \multirow{2}{*}{ Porcentaje de población urbana } & PAC & 81,32 & 27,48 \\
\hline & PLN & 33,29 & 30,84 \\
\hline \multirow{2}{*}{ Tasa neta de participación } & PAC & 55,64 & 3,78 \\
\hline & PLN & 47,59 & 7,67 \\
\hline \multirow{2}{*}{ Tasa de ocupación } & PAC & 53,99 & 3,67 \\
\hline & PLN & 46,75 & 5,20 \\
\hline \multirow{2}{*}{ Tasa de desempleo abierto } & PAC & 2,96 & 0,83 \\
\hline & PLN & 3,16 & 1,42 \\
\hline \multirow{2}{*}{ Porcentaje de población económicamente inactiva } & PAC & 44,36 & 3,78 \\
\hline & PLN & 51,74 & 5,24 \\
\hline \multirow{2}{*}{ Relación de dependencia económica } & PAC & 1,34 & 0,22 \\
\hline & PLN & 1,88 & 0,38 \\
\hline
\end{tabular}

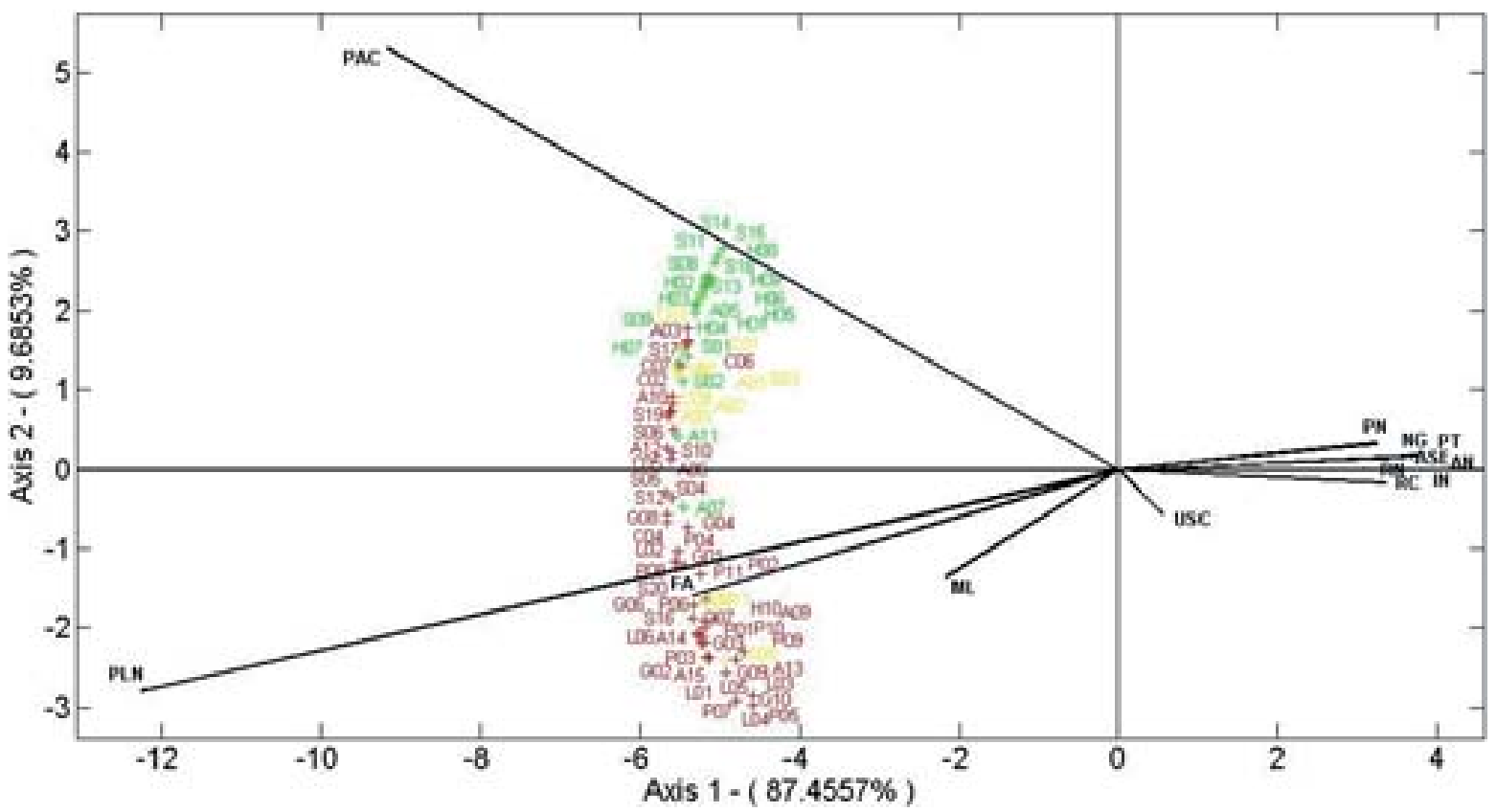

Gráfico 3. Representación de las elecciones Presidenciales según partido político e Índice de Desarrollo Social de los cantones. 
Al considerar las variables socioeconómicas se descubren las diferencias estadísticas entre los cantones ganados por el PAC y PLN. El PAC obtuvo más votos en los cantones caracterizados por mayor tasa neta de participación $(56,0)$, con una tasa de ocupación $(54,3)$ y tienen más población urbana $(82,6)$. En tanto, el PLN ganó en los cantones con mayor porcentaje de población económicamente inactiva $(51,2)$ y tienen una tasa de fecundidad general de 66,3. Debe considerarse que la tasa de fecundidad está relacionada con el nivel de educación de las madres e ingresos económicos. Al respecto, las madres con menor ingreso económico y bajos niveles de escolaridad tienden a incrementar la probabilidad de tener más cantidad de hijos (Cuadro 3).

La representación Biplot del resultado de las elecciones por provincia, tiene una bondad de ajuste global para el primer plano factorial (ejes 1 y 2 ) de 99,2\% (Gráfico 4). Primero, se observa que por el Partido Acción Ciudadana (PAC) votaron mayoritariamente las provincias de Alajuela, de Cartago, de San José y de Heredia. En tanto, los electores de las provincias de Puntarenas, de Limón y de Guanacaste, tendieron a votar por el Partido Liberación Nacional (PLN) y Frente Democrático (FA). Segundo, al considerar el Índice de Desarrollo Social (IDS) de las provincias, es interesante notar que, en la provincia de mejor IDS, como Heredia, los electores se inclinaron votar por PAC. En San José que tiene un IDS medio, los votantes también apoyaron a este partido. Las demás provincias con IDS bajos apoyaron al PAC, PLN y FA.

\begin{tabular}{|c|c|c|c|}
\hline \multicolumn{4}{|c|}{$\begin{array}{r}\text { Cuadro 3. Comparación de los promedios aritn } \\
\text { variables socioeconómicas según cantones gar } \\
\text { Partido Acción Ciudadana (PAC) } \\
\text { y Partido Liberación Nacional }\end{array}$} \\
\hline \multicolumn{2}{|c|}{ Variable } & \multirow{2}{*}{$\begin{array}{c}\text { Media } \\
56,0\end{array}$} & $\begin{array}{c}\text { Desviación } \\
\text { estándar }\end{array}$ \\
\hline \multirow{2}{*}{ Tasa neta de participación } & PAC & & 3,077 \\
\hline & PLN & 48,8 & 3,384 \\
\hline \multirow{2}{*}{ Tasa de ocupación } & PAC & 54,3 & 2,982 \\
\hline & PLN & 47,2 & 3,298 \\
\hline \multirow{2}{*}{$\begin{array}{l}\text { Porcentaje de población } \\
\text { económicamente inactiva }\end{array}$} & PAC & 44,0 & 3,077 \\
\hline & PLN & 51,2 & 3,384 \\
\hline \multirow{2}{*}{$\begin{array}{l}\text { Porcentaje de población } \\
\text { urbana }\end{array}$} & PAC & 82,6 & 21,444 \\
\hline & PLN & 46,1 & 19,752 \\
\hline \multirow{2}{*}{ Tasa de fecundidad general } & PAC & 52,9 & 6,825 \\
\hline & PLN & 66,3 & 13,673 \\
\hline
\end{tabular}

La representación Biplot del resultado de las elecciones por provincia, tiene una bondad de ajuste global para el primer plano factorial (ejes 1 y 2 ) de 99,2\% (Gráfico 4). Primero, se observa que por el Partido Acción Ciudadana (PAC) votaron mayoritariamente las provincias de Alajuela, de Cartago, de San José y de Heredia. En tanto, los electores de las provincias de Puntarenas, de Limón y de Guanacaste, tendieron a votar por el Partido Liberación Nacional (PLN) y Frente Democrático (FA). Segundo, al considerar el Índice de Desarrollo Social (IDS) de las provincias, es interesante notar que, en la provincia de mejor IDS, como Heredia, los electores se inclinaron votar por PAC. En San José que tiene un IDS medio, los votantes también apoyaron a este partido. Las demás provincias con IDS bajos apoyaron al PAC, PLN y FA. 


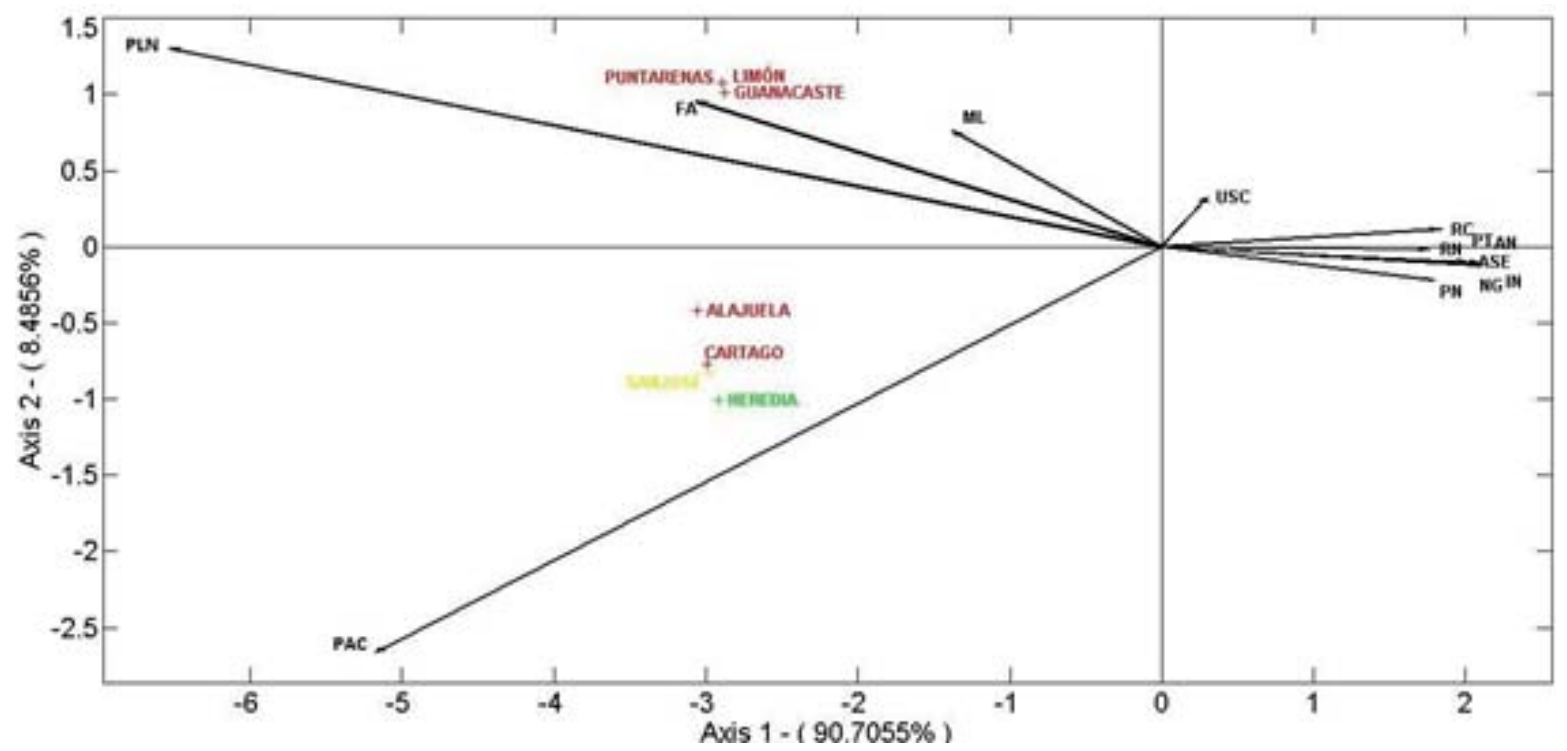

Gráfico 4. Representación de las elecciones Presidenciales según partido político e Índice de Desarrollo Social de los cantones.

Finalmente, es necesario resaltar que los vectores con dirección al Partido Acción Ciudadana (AC) y Partido Liberación Nacional (LN) son extensos, indicando que ambos partidos presentaron una gran variabilidad en los votos obtenidos por provincia. Se visualizan dos conglomerados, esto debido a que la distancia entre ellos es pequeña. Grupo 1: Alajuela, Cartago, San José y Heredia. Grupo 2: Puntarenas, Limón y Guanacaste. Los partidos pequeños se concentran alrededor del eje horizontal indicando que estuvieron a su favor pequeñas cantidades de votos.

\section{Conclusiones}

El estudio nos permite concluir que el número de votos obtenidos por el Partido Acción Ciudadana presenta una correlación positiva con las variables Índice de Desarrollo Social y porcentaje de población urbana. Igual resultado se presentó con la tasa neta de participación $(r=0,47)$ y tasa de ocupación $(\mathrm{r}=0,45)$. Esto implica que regiones con mayores tasas de participación y tasa de ocupación tendieron a apoyar al Partido Acción Ciudadana.

Se determinó que hay diferencias estadísticamente significativas a un nivel de confiabilidad del 95\% entre los distritos ganados por los partidos Acción Ciudadana y Liberación Nacional según variables demográficas y económicas. El Partido Acción Ciudadana obtuvo la mayor cantidad de votos a favor en los distritos con las siguientes características: tienen mayor porcentaje de población urbana $(81,3)$, la tasa neta de participación es 55,6 y la tasa de ocupación es 54,0. El Partido Liberación ganó los distritos con mayores problemas socioeconómicos, caracterizados por una relación de dependencia demográfica y de dependencia infantil de 52 y 41 respectivamente.

El análisis estadístico aplicando el método Biplot se ha descubierto como una herramienta multivariada útil para estudiar los determinantes del voto en Costa Rica en la elección presidencial de 2014, ya que ha permitido caracterizar el fenómeno político nacional. En este sentido, los electores votaron no solamente influenciados por la propaganda política, sino que, se ven estimulados, además, por la tendencia de variables tales como el desempleo, tasa neta de participación, índice de desarrollo Social, etc.

Por lo anterior, se puede determinar la verdad científica de las explicaciones sencillas de que las zonas costeras tienden a votar por el Partido 
Liberación Nacional, lo cual significa que en realidad lo que está implícito, es las desigualdades económicas y las brechas políticas que se manifiestan geográficamente. De hecho, el Partido Acción Ciudadana obtuvo la victoria gracias a los votos de la Gran Área Metropolitana, donde las condiciones socioeconómicas son relativamente mejores y donde se concentra la mayor cantidad de la población.

\section{Bibliografía}

Araya, C. (2015). "Evaluación del estado socioeconómico del cantón de San Ramón”. Revista Pensamiento Actual, 15(24), 73-81. - (2015) "Análisis de los resultados para la elección de diputados y de la quiebra del voto en Costa Rica 2014". Revista Intersedes, 16(33), 40-52.

Instituto Nacional de Estadística y Censos de Costa Rica (INEC). (2011). Censo de Población y Vivienda.

Ministerio de Planificación Nacional y Política Económica. (2013). Costa Rica: Índice de Desarrollo Social.

Moreno, A. (2009).La decisión electoral, votantes, partidos y democracia en México. México: Miguel Ángel Porrúa

Vicente, J. (2010) MULTBIPLOT: A package for Multivariate Analysis using Biplots. Departamento de Estadística. Universidad de Salamanca, España. Recuperado de: http:// biplot.usal.es/ClassicalBiplot/index.html

Urcuyo, C. (2015). “De la alegría a la decepción”. La Nación: 27 de abril del 2015. Recuperado de: http://www.nacion.com/opinion/ foros/Constantino- Urcuyo-alegriadecepcion_0_1484051587.html 